\title{
Usefulness of neutrophil-to-lymphocyte ratio and platelet-to-lymphocyte ratio as predictors of severity on Fournier fasciitis of the Hospital General de México "Dr. Eduardo Liceaga"
}

\author{
Agustín Guemes-Quinto ${ }^{1}$, Ansony R. Godínez-Vidal ${ }^{1 *}$, Juan A. Villanueva-Herrero ${ }^{2}$, Billy Jimenez-Bobadilla ${ }^{2}$, \\ Sergio U. Perez Escobedo ${ }^{1}$, Hamzeh Bandeh-Moghaddam ${ }^{2}$ and Noé I. Gracida-Mancilla ${ }^{1}$ \\ ${ }^{1}$ Departamento de Cirugía General; ${ }^{2}$ Servicio de Colon, Recto y Ano. Hospital General de México "Dr. Eduardo Liceaga”, Mexico City, Mexico
}

\begin{abstract}
Background: Fournier fasciitis (FF) severity is traditionally assessed by the Fournier gangrene severity index (FGSI), as it is an internationally accredited index, which consists of some clinical and laboratory parameters. However, the neutrophil-to-lymphocyte ratio (NLR) and platelet-to-lymphocyte ratio (PLR) have shown to be useful as predictors of severity in different septic and ischemic processes, and a full blood count is sufficient for its application. Objectives: The objectives of this study were to determine if there is a correlation between FF severity measured by NLR and PLR, and the severity measured by the FGSI scale. Materials and methods: We conducted a retrospective, observational, and analytical study to evaluate the usefulness of the NLR and PLR as indicators of severity on Fournier fasciitis. Results: We included 45 cases treated at Hospital General de México "Dr. Eduardo Liceaga", from 2010 to 2016. Forty cases were of anorectal origin and five of them of urological origin. The severity assessment scale was set for FGSI at 9 points, NLR at 7 points, and PLR at 140 points. About $60 \%$ of cases deemed severe by NLR, resulted in an FGSI score lower than $9, p=0.547$, without statistical significance. About $72 \%$ of cases diagnosed as severe by PLR, yielded an FGSI score lower than 9, $p=0.613$, without statistical significance. Conclusions: Additional studies would be required to confirm or rule out these results.
\end{abstract}

Key words: Neutrophil-to-lymphocyte ratio. Platelet-to-lymphocyte ratio. Fournier gangrene severity index. Severity.

\section{Introduction}

Fournier fasciitis (FF) is a serious infectious and life-threatening disease of rapid dissemination. Therefore, it is actually worth of early diagnosis and timely management. A polymicrobial pathology affects the genital, perineal, and perianal region ${ }^{1}$. It is named after Jean Alfred Fournier, a French dermatologist, who presented the case of a young man with perineal gangrene in $1883^{2}$. Due to its acute and rapid progression and polymicrobial involvement, Fournier's gangrene continues to represent high mortality rates despite advances in surgical techniques, critical care, and the development of new antibiotics. Most studies report mortality rates between $20 \%$ and $40 \%$, with a range of $4-88 \% 3$.

Different scoring systems and prognostic factors have been proposed in an effort to predict FF survival and prognosis. The Fournier gangrene severity
Correspondence:

*Ansony R. Godínez-Vidal

E-mail: ansony.rgv@gmail.com
Available online: $17-10-2019$ Rev Med Hosp Gen Mex. 2019;82(4):175-178 www.hospitalgeneral.mx under the CC BY-NC-ND license (http://creativecommons.org/licenses/by-nc-nd/4.0/). 
Table 1. Fournier gangrene severity index (adapted from Laor et al. ${ }^{4}$ )

\begin{tabular}{|c|c|c|c|c|c|c|c|c|c|}
\hline \multirow[t]{2}{*}{ Variables/assigned points } & \multicolumn{4}{|c|}{ High } & \multirow{2}{*}{$\frac{\text { Normal }}{0}$} & \multicolumn{4}{|c|}{ Low } \\
\hline & +4 & +3 & +2 & +1 & & +1 & +2 & +3 & +4 \\
\hline Temp. ${ }^{\circ} \mathrm{C}$ & $>41$ & $39-40.9$ & - & $38.5-38.9$ & $36-38.4$ & $34-35.9$ & $32-33.9$ & $30-31.9$ & $<39.9$ \\
\hline Heart rate & $>180$ & $140-179$ & $110-139$ & - & $70-109$ & - & $55-69$ & $40-54$ & $<39$ \\
\hline Respiratory rate & $>50$ & $35-49$ & - & $25-34$ & $12-24$ & $10-11$ & $6-9$ & - & $<5$ \\
\hline Serum sodium mmol/l & $>180$ & $160-179$ & $155-159$ & $150-154$ & $130-149$ & - & $120-129$ & $111-119$ & $<110$ \\
\hline Serum potassium mmol/l & $>7$ & $6-6.9$ & - & $5.5-5.9$ & $3.5-5.4$ & 3-3.4 & $2.5-2.9$ & - & $<2.5$ \\
\hline Serum creatinine $\mathrm{mg} / 100 \mathrm{ml}$ & $>3.5$ & $2-3.4$ & $1.5-1.9$ & - & $0.6-1.4$ & - & $<0.6$ & - & - \\
\hline Hematocrit (HT) & $>60$ & - & $50-59.9$ & $46-49.9$ & $30-45.9$ & - & $20-29.9$ & - & $<20$ \\
\hline Leukocytes total $/ \mathrm{mm}^{3} \times 1000$ & $>40$ & - & $20-39.9$ & $15-19.9$ & $3-14.9$ & - & $1-2.9$ & - & $<1$ \\
\hline Venous serum bicarbonate $\mathrm{mmol} / \mathrm{l}$ & $>52$ & $41-51.9$ & - & $32-40.9$ & $22-31.9$ & - & $18-21.9$ & $15-17.9$ & $<15$ \\
\hline
\end{tabular}

index (FGSI) (Table 1), first described in 1995, is a scoring system that consists of some clinical and laboratory parameters that help predict prognosis and survival ${ }^{4}$.

FF severity is traditionally evaluated by means of the FGSI scale, which is an internationally accredited index, and requires hematic biometrics, blood chemistry, serum electrolytes and blood gas test, neutrophil-to-lymphocyte ratio (NLR), and platelet-to-lymphocyte ratio $(P L R)$ for its application. These items have proven to be useful as predictors of severity in different septic and ischemic processes; in addition, hematic biometrics may be the only requirement for its application ${ }^{5,6}$.

Given the simplicity of determining these ratios, their utility to measure FF severity should be evaluated by comparing them against the usual FGSI measurement.

\section{Materials and methods}

A retrospective, observational, analytical study of patients diagnosed with FF was performed to determine if the severity measured by the FGSI scale is associated with the severity measured by the NLR and PLR ratios. Patients diagnosed with FF and treated at the Hospital General de México "Dr. Eduardo Liceaga" from 2010 to 2016 were included in the study.

\section{Results}

A sample of 45 cases was included from 2010 to $2016,40(88.8 \%)$ were male and 5 of them (11.2\%) were
Table 2. Severity values for the sample

\begin{tabular}{|l|c|c|}
\hline Index & Mean & SD \pm \\
\hline FGSI & 7.7 & 3.8 \\
\hline NLR & 13.3 & 10.4 \\
\hline PLR & 215 & 170 \\
\hline
\end{tabular}

FGSI: Fournier gangrene severity index; NLR: neutrophil-to-lymphocyte ratio; PLR: platelet-to-lymphocyte ratio.

female. The average age of the sample was 50.6 years. In 40 cases, the FF origin was anorectal and in five cases, the origin was urological.

Regarding the severity scales under evaluation, a mean score for the FGSI sample of 7.7 (SD 3.8) was found. A mean NLR score of 13.3 (SD 10.4) and a PLR of 215 points (SD 170) were obtained, as shown in table 2 .

The severity assessment scale was set for FGSI values at 9 points, NLR at 7 points, and PLR at 140, which are the values referred to in literature ${ }^{7-9}$. By categorizing the severity, the values were obtained, which are shown in table 3.

About $40 \%$ of cases deemed severe by the NLR were also considered severe by the FGSI, while $60 \%$ of cases diagnosed as severe by the NLR resulted in an FGSI score lower than 9. When applying Fisher's exact test, a statistically non-significant $p=0.077$ was obtained. By submitting these differences to statistical hypothesis testing, using Mann-Whitney U-test, for independent samples, we obtained $p=0.547$, without statistical significance (Table 4). When comparing the severity 
Table 3. Variable categorization according to the level of severity

\begin{tabular}{|l|c|c|}
\hline Variable & Mild & Severe \\
\hline FGSI & $<9(66.7 \%)$ & $10(33.3 \%)$ \\
\hline NLR & $<7(22.2 \%)$ & $8(77.8 \%)$ \\
\hline PLR & $<140(44.4)$ & $141(56.6 \%)$ \\
\hline
\end{tabular}

FGSI: Fournier gangrene severity index; NLR: neutrophil-to-lymphocyte ratio; PLR: platelet-to-lymphocyte ratio.

Table 4. FGSI versus NLR

\begin{tabular}{|l|c|c|c|c|}
\hline \multirow{2}{*}{} & \multicolumn{2}{|c|}{ Severity by FGSI } & \multirow{2}{*}{ Total } \\
\cline { 3 - 4 } & Severe $>\mathbf{1 0}$ & Mild $<\mathbf{9}$ & \\
\hline $\begin{array}{l}\text { Severity } \\
\text { by NLR }\end{array}$ & Severe $>8$ & 14 & 21 & 35 \\
\hline & Mild $<7$ & 1 & 9 & 10 \\
\hline & & $10.0 \%$ & $90.0 \%$ & $100.0 \%$ \\
\hline Total & 15 & 30 & 45 \\
\hline
\end{tabular}

FGSI: Fournier gangrene severity index; NLR: neutrophil-to-lymphocyte ratio.

Table 5. FGSI versus PLR

\begin{tabular}{|l|c|c|c|c|}
\hline \multirow{2}{*}{} & \multicolumn{2}{|c|}{ Severity by FGSI } & \multirow{2}{*}{ Total } \\
\cline { 3 - 4 } & Severe $>\mathbf{1 0}$ & Mild $<\mathbf{9}$ & \\
\hline $\begin{array}{l}\text { Severity } \\
\text { by PLR }\end{array}$ & Severe $>141$ & 7 & 18 & 25 \\
\hline & Mild $<140$ & 8 & 12 & 20 \\
\hline & & $40.0 \%$ & $60.0 \%$ & $100.0 \%$ \\
\hline Total & 15 & 30 & 45 \\
\hline
\end{tabular}

FGSI: Fournier gangrene severity index; NLR: neutrophil-to-lymphocyte ratio.

determined by the PLR with the severity determined by $\mathrm{FGSI}$, it is observed that $28 \%$ of the cases diagnosed as severe by the PLR corresponded to an FGSI score $>10$, while $72 \%$ diagnosed as severe by the PLR, yielded an FGSI score lower than 9. While applying the Chi-square test as a test statistic, $p=0.396$ was obtained, without statistical significance. By submitting these differences to the Mann-Whitney U-test, we obtained $p=0.613$, without statistical significance (Table 5).

\section{Discussion}

FF management is complex and requires a timely evaluation in the emergency services to apply the appropriate treatment as soon as possible. The systematic use of prediction scales overtime has allowed to improve the prognosis of patients by making a prediction of severity and mortality and based thereon, to establish the ideal treatment. The clearest example is the FGSI scale, which is accepted in multiple studies. The FGSI scale requires the calculation of numerous variables, which are not always accessible in all media. In an attempt to simplify the initial evaluation, studies have been conducted using scales such as LRINEC, UFGSI, sAPGAR, and Charlson Comorbidity Index. The NLR and PLR are two markers that have been tested in different pathologies, including FF, and which have yielded very variable results. Studies conducted for this pathology have attempted to simplify the way to assess the clinical stage of the disease with a single laboratory study. No previous studies were found in Mexico. Kahramanaca reports in a study with 68 patients that high NLR and PLR are associated with the need for more debridement, higher hospitalization costs, and higher mortality rates ${ }^{7}$. Manu in a study with 24 patients reported higher morbidity and mortality rates with high FGSI, NLR, and PLR values ${ }^{8}$.

Yim et al. also reported NLR and PLR as independent prognostic factors that are associated with increased mortality from $\mathrm{FF}^{9}$.

In this study, there was no statistically significant relationship while comparing NLR and PLR values with the FGSI scale, which correlated the severity measured between the different means to determine the severity of FF. It is worth noting that in the present sample obtained for convenience, there were no mortality cases. The reference values for NLR and PLR have not been established uniformly and do not allow establishing a position to determine the ideal treatment at the time of diagnosis.

\section{Conclusions}

FF severity, measured by the NLR and the PLR, does not correspond to the severity measured by FGSI. A case and control observation study should be undertaken that includes cases with and without mortality so that a more adequate conclusion can be reached.

\section{Conflicts of interest}

The authors declare that there are no conflicts of interest. 


\section{Ethical disclosures}

Protection of human and animal subjects. The authors declare that no experiments were performed on humans or animals for this study.

Confidentiality of data. The authors declare that they have followed the protocols of their work center on the publication of patient data.

Right to privacy and informed consent. The authors declare that no patient data appear in this article.

\section{References}

1. Morpurgo E, Galandiuk S. Fournier's gangrene. Surg Clin North Am. 2002;82:1213-24.

2. Fournier JA. Gangrene foudroyante de la verge. Semin Med. 1883;3:345.
3. Sorensen MD, Krieger JN, Rivara FP, Klein MB, Wessells H. Fournier's gangrene: management and mortality predictors in a population based study. J Urol. 2009;182:2742-7.

4. Laor E, Palmer LS, Tolia BM, Reid RE, Winter HI. Outcome prediction in patients with Fournier's Gangrene. J Urol. 1995;154:89-92.

5. Ishizuka M, Shimizu T, Kubota K. Neutrophil-to-lymphocyte ratio has a close association with gangrenous appendicitis in patients undergoing appendectomy. Int Surg. 2012:97:299-304.

6. Azab B, Shah N, Akerman M, McGinn JT Jr. Value of platelet/lymphocyte ratio as a predictor of all-cause mortality after non-ST-elevation myocardial infarction. J Thromb Thrombolysis. 2012;34:326-34.

7. Kahramanca S, Kaya O, Özgehan G, Irem B, Dural I, Küçükpınar T, et al. Are neutrophil-lymphocyte ratio and platelet-lymphocyte ratio as effective as Fournier's Gangrene severity index for predicting the number of debridements in Fourner's Gangrene? Ulus Travma Acil Cerrahi Derg. 2014;20:107-12.

8. Manu MA, Parlitenau B, Priel I, Dudu C, Surcel C, Stefan B. Neutrophil-lymphocyte ratio and platelet-lymphocyte ratio vs. Fournier Gangrene severity index as prognostic factors in management of Fournier Gangrene. Eur Urol Suppl. 2015;14:e1171

9. Yim SU, Kim SW, Ahn JH, Cho YH, Chung H, Hwang EC, et al. Neutrophil to lymphocyte and platelet to lymphocyte ratios are more effective than the Fournier's Gangrene severity index for predicting poor prognosis in Fournier's Gangrene. Surg Infect (Larchmt). 2016; 17:217-23 